\title{
Self-Assembled Nanostructuring of a-Si:H Films with Ultrashort Light Pulses
}

\author{
Mindaugas Gecevičius ${ }^{1 *}$, Martynas Beresna ${ }^{1}$, Andrey G. Kazanskii ${ }^{2}$, Peter G. Kazansky ${ }^{1}$ \\ ${ }^{1}$ Optoelectronics Research Centre, University of Southampton, SO17 1BJ, United Kingdom \\ ${ }^{2}$ Physics Department, M.V. Lomonosov Moscow State University, Moscow, Russia \\ mg2e10@orc.soton.ac.uk
}

For several decades, hydrogenated amorphous silicon (a-Si:H) has been playing a significant role in the world's production of photovoltaic modules. In this work, we investigate different types of modifications induced by a femtosecond laser in a-Si:H thin films. We demonstrate that several distinctive modification regimes with peculiar optical properties can be obtained in a narrow range of the laser pulse energies.

The experiments were performed with the PHAROS (LightConversion Ltd.) femtosecond laser system operating at $1030 \mathrm{~nm}$ with a pulse duration of $300 \mathrm{fs}$ at a $200 \mathrm{kHz}$ repetition rate. The laser beam was focused with an aspheric lens $(\mathrm{NA}=0.16) 80 \mu \mathrm{m}$ above the surface of the $300 \mathrm{~nm}$ thickness a-Si:H film deposited onto fused silica glass. A uniform modified area was formed by laser tracks written at $0.5 \mathrm{~mm} / \mathrm{s}$ and $5 \mathrm{~mm} / \mathrm{s}$ speeds with a $2 \mu \mathrm{m}$ step. The pulse energy was gradually increased for each laser track along the structure (Fig. 1). The sample was analysed with an Olympus BX51 optical microscope and a quantitative birefringence measurement system CRi Abrio.
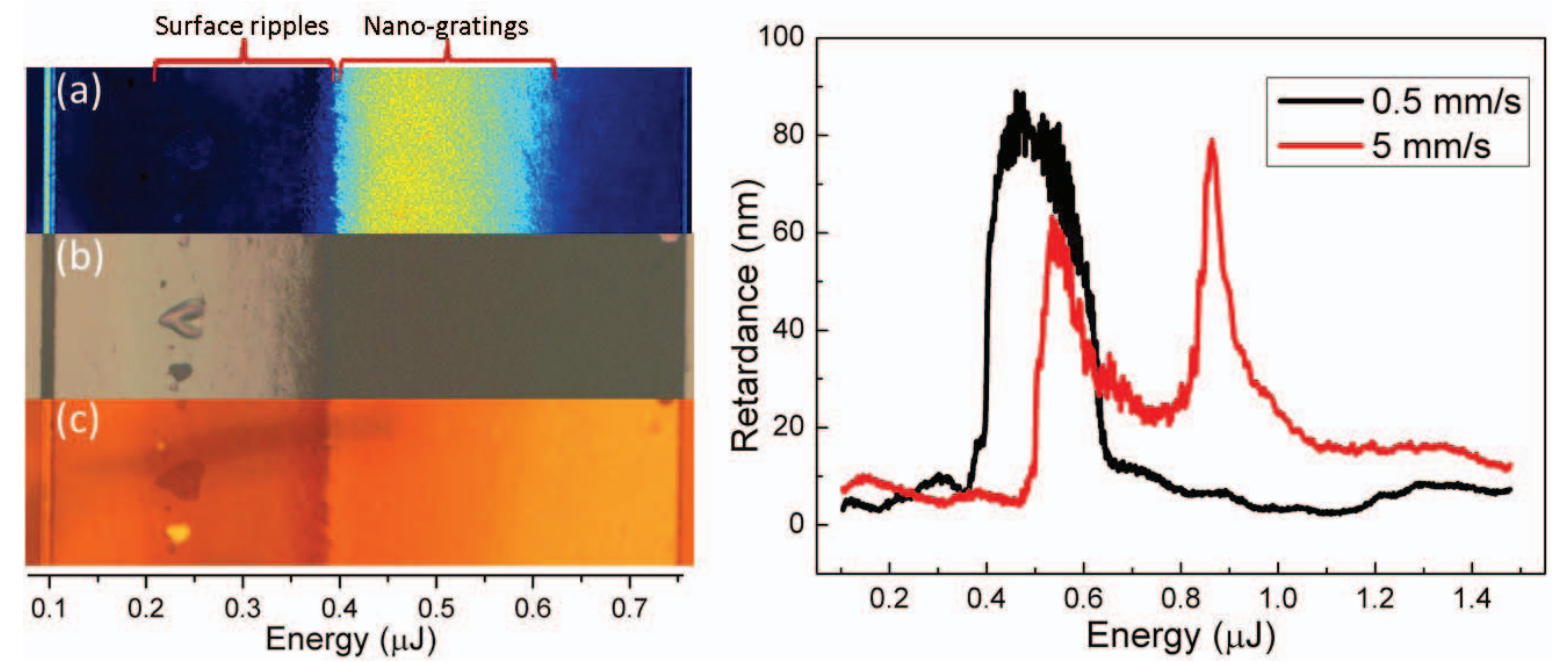

Fig. 1 Microscopic images of a-Si:H modified with a femtosecond laser $(0.5 \mathrm{~mm} / \mathrm{s}$ writing speed $)$ under different pulse energies (left). Scale indicates pulse energy in $\mu \mathrm{J}$. Different imaging modes reveal different features of the same modified region. Image (a) shows retardance distribution. Images (b) and (c) are respectively in reflection and transmission microscopy modes. Retardance dependence on laser pulse energy for two different writing speeds (right).

Depending on the laser pulse energy, several regimes can be distinguished (Fig. 1). The dark area (Fig. 1(c)), from $0.2 \mu \mathrm{J}$ to $0.4 \mu \mathrm{J}$, is the region where the crystallization of a-Si:H film occurs as indicated by the narrowing of the Raman spectrum [1]. At around $0.23 \mu \mathrm{J}$, the film is partially removed by the spallation process (Fig. 1(b), (c)) [2]. At $\sim 0.25 \mu \mathrm{J}$, the spallation process is terminated and the film is again modified homogeneously. From $\sim 0.4 \mu \mathrm{J}$, the laser irradiation starts oxidising the a-Si:H film. As the energy increases further, the sample becomes more transparent. Simultaneously, the film becomes strongly birefringent (Fig. 1(a)), which indicates subwavelength periodic structure formation oriented perpendicularly to the polarization plane. Closer inspection revealed that ripples parallel to polarization plane are also formed at the amorphous silicon crystallization regime. The observed birefringence $\Delta n=n_{\mathrm{e}}-n_{\mathrm{o}}$ is more than ten times larger than in fused silica (Fig. 1 (right)) [3]. Dichroism is also observed at this regime. Retardance is strongly dependent on the pulse energy, at the beginning of birefringent modification zone and then gradually reduces to background noise level. After the birefringence disappears, the transparency of the modified film increases till the a-Si:H is fully converted into $\mathrm{SiO}_{2}$. Peculiarly, at higher writing speeds $(5 \mathrm{~mm} / \mathrm{s})$ in the same range of pulse energies, two birefringent regions can be observed.

Strong birefringence and dichroism of a-Si:H films induced by ultrafast laser nanostructuring can be used for integrated spatially variant polarization optics.

\section{References}

[1] A.V. Emelyanov et al.,"Visible luminescence from hydrogenated amorphous silicon modified by femtosecond laser radiation," Appl. Phys. Lett. 101, 081902 (2012).

[2] J. Bovatsek et al., "Thin film removal mechanisms in ns-laser processing of photovoltaic materials," Thin Solid Films 518, 2897 (2010). [3] E. Bricchi et al., "Form birefringence and negative index change created by femtosecond direct writing in transparent materials," Opt. Lett. 29, 119 (2004). 